\title{
Using quantum optical sensors for determining the Earth's gravity field from space
}

\author{
Jürgen Müller ${ }^{1} \cdot \mathrm{Hu} \mathrm{Wu}^{1}$ (D)
}

Received: 15 August 2019 / Accepted: 13 July 2020 / Published online: 24 July 2020

(c) The Author(s) 2020

\begin{abstract}
Quantum optical technology provides an opportunity to develop new kinds of gravity sensors and to enable novel measurement concepts for gravimetry. Two candidates are considered in this study: the cold atom interferometry (CAI) gradiometer and optical clocks. Both sensors show a high sensitivity and long-term stability. They are assumed on board of a low-orbit satellite like gravity field and steady-state ocean circulation explorer (GOCE) and gravity recovery and climate experiment (GRACE) to determine the Earth's gravity field. Their individual contributions were assessed through closed-loop simulations which rigorously mapped the sensors' sensitivities to the gravity field coefficients. Clocks, which can directly obtain the gravity potential (differences) through frequency comparison, show a high sensitivity to the very long-wavelength gravity field. In the GRACE orbit, clocks with an uncertainty level of $1.0 \times 10^{-18}$ are capable to retrieve temporal gravity signals below degree 12 , while $1.0 \times 10^{-17}$ clocks are useful for detecting the signals of degree 2 only. However, it poses challenges for clocks to achieve such uncertainties in a short time. In space, the CAI gradiometer is expected to have its ultimate sensitivity and a remarkable stability over a long time (measurements are precise down to very low frequencies). The three diagonal gravity gradients can properly be measured by CAI gradiometry with a same noise level of $5.0 \mathrm{mE} / \sqrt{\mathrm{Hz}}$. They can potentially lead to a 2-5 times better solution of the static gravity field than that of GOCE above degree and order 50, where the GOCE solution is mainly dominated by the gradient measurements. In the lower degree part, benefits from CAI gradiometry are still visible, but there, solutions from GRACE-like missions are superior.
\end{abstract}

Keywords Quantum optical sensors · Optical clocks · Relativistic geodesy · Atomic gradiometry · Gravity field

\section{Introduction}

The determination of a precise gravity field model is essential for a variety of geoscience applications, such as monitoring global sea level rise, refining ocean circulation models, realizing a global height reference system, understanding the Earth's geodynamics and so on (Pail et al. 2015). In the past, the successful application of satellite gravity missions, like GRACE (Tapley et al. 2004) that is now continued by GRACE-FO (GRACE Follow-On) (Kornfeld et al. 2019), and GOCE (Pail et al. 2011), has remarkably advanced our knowledge of the Earth's gravity field. Now, ongoing efforts are dedicated to further improve the performance of future missions, which include the upgrade of onboard sensors,

$\mathrm{Hu} \mathrm{Wu}$

wuhu@ife.uni-hannover.de

1 Institut für Erdmessung (IfE), Leibniz Universität Hannover, Schneiderberg 50, 30167 Hannover, Germany e.g., the ranging system using laser interferometry (Sheard et al. 2012), the consideration of more satellite pairs, e.g., the Bender satellite formation (Elsaka et al. 2014), and the improvement of background models that are mainly needed to reduce high-frequency atmospheric and ocean mass contributions (Dobslaw et al. 2016).

Meanwhile, quantum optical technology that has significantly advanced in the past years provides the opportunity to develop new kinds of gravity sensors and to enable novel measurement concepts for gravimetry. One promising sensor is the CAI gradiometer. It was proposed to measure gravity gradients using spatially separated interferometers with free falling atoms (Snadden et al. 1998). This instrument is fundamentally different from other types of gradiometers as its proof masses are individual atoms (clouds of atoms) rather than precisely machined macroscopic objects. It has a long-term stability, less affected by drifts, and achieves a sensitivity level of a few tens of $\mathrm{E} / \sqrt{\mathrm{Hz}}$ on ground (Sorrentino et al. 2014) or $3 \mathrm{E} / \sqrt{\mathrm{Hz}}$ (Asenbaum et al. 2017). A signifi- 
cant improvement of the sensitivity can be expected when the instruments were operated in space. The "free fall" condition allows much longer interrogation times for atom interferometry, i.e., a few tens of seconds versus hundreds of ms on ground (the sensitivity can principally be improved with the square of the interrogation time), and the "quiet" environment can reduce perturbations (e.g., the seismic noise). It is estimated that the CAI gradiometer can achieve the level of $\mathrm{mE} / \sqrt{\mathrm{Hz}}$ in space, and some studies predict that the sensitivity could even go down to $0.07 \mathrm{mE} / \sqrt{\mathrm{Hz}}$ (Rakholia et al. 2017)

Another candidate that receives considerable attention is the optical clock. It can provide the frequency information with a very high accuracy. Using optical clocks at different locations, it is possible to observe the tiny frequency change that is caused by the gravitational potential difference (gravitational redshift effect). This concept was introduced for retrieving the Earth's gravitational potential difference between two sites through the comparison of clocks' frequencies (Bjerhammar 1985, 1986). The obtained potential difference can be straightforwardly scaled to a physical height difference (Vermeer 1983). In geodesy, gravity potential values are used as so-called geopotential numbers which are the core of any physical height (Torge and Müller 2012; Denker et al. 2018). Today, the latest generation of optical clocks achieve a fractional frequency uncertainty of $1.0 \times 10^{-18}$ and even beyond (McGrew et al. 2018; Brewer et al. 2019) that approximates to $0.1 \mathrm{~m}^{2} / \mathrm{s}^{2}$ in terms of potential and $1.0 \mathrm{~cm}$ in terms of height. Therefore, it is very interesting for geodesy as Global Geodetic Observing System (GGOS) demands $1 \mathrm{~mm}$ accuracy for positions as a central goal (Plag et al. 2009). For physical heights, the next step would be achieving a consistent $1 \mathrm{~cm}$ accuracy level. Clocks are thus considered as a new powerful measurement tool and are relevant for a variety of geodetic applications (Müller et al. 2018; Delva et al. 2019).

Both sensors are promising candidates for future satellite gravity missions. Their potential for improving the Earth's gravity field has gained great interests in physics, geodesy and Earth science communities. In this paper, we quantitatively evaluate the benefit of them for the determination of different parts of the Earth's gravity field. We map the sensitivity of both individual sensors to the gravity field coefficients through closed-loop simulations. The simulator takes synthesized realistic observations along a well-defined satellite orbit (e.g., the GOCE and GRACE orbit) as input and estimates the gravity field coefficients using a rigorous least-squares adjustment. In addition, a joint solution was recovered from both kinds of observations with proper weighting factors, which combines the contribution of each sensor. To evaluate the results, the coefficient differences between the recovered and the reference models as well as the formal errors (standard deviations of the estimated gravity field coefficients) are taken to calculate different numerical quantities like degree medians and degree variances.

This paper is organized as follows: The quantum optical gravity sensors and their relevant measurement principles are reviewed in Sect. 2. Next, the closed-loop simulator and the datasets are described. The recovered gravity field solutions are presented and discussed in Sect. 4. The concluding remarks and future perspectives are addressed in the last section.

\section{Quantum optical sensors for geodesy}

Quantum optics is one of the frontier fields of scientific research. It has been widely accepted that the development of quantum optics may bring revolutionary changes in the fields of computing, communication and image sensing (Browne et al. 2017). Besides, another important application of quantum optics is for precision measurement. The wave property of atoms allows to use coherence of atomic pulses for the measurements of inertial accelerations, gravity and rotation (Kitching et al. 2011; Barrett et al. 2016). Developments in quantum technology could lead to a breakthrough for the next generation of accelerometers, gradiometers and gyroscopes. Another high-precision sensor is the optical clock. It is the most precise device for providing ultra-high frequencies. Among these sensors, the atomic gradiometer and optical clocks are most relevant for our topic of gravity field determination.

\subsection{CAl gradiometer}

Gravity gradiometry measures the second-order partial derivatives of the Earth's gravity potential. It is sensitive to tiny variations of gravity and capable to map the fine structure of gravity field down to small spatial scales. The first spacebased gravity gradiometry was implemented in the GOCE mission (Rummel et al. 2011), with an extreme low satellite orbit. During the more than 4 years' lifetime, GOCE delivered hundreds of millions of gravity gradients. These observations have greatly improved the performance of the global gravity field model, especially in the medium- and short-wavelength parts (Brockmann et al. 2014; Bruinsma et al. 2014). To give a few examples, the improved gravity field is useful to refine ocean circulation models (Bingham et al. 2011) and the ocean dynamic topography (Becker et al. 2014). In addition, the GOCE gravity gradients provide valuable information for deriving a global Moho surface (Reguzzoni et al. 2013) and yielding insights into space weather (Ince and Pagiatakis 2016).

The GOCE gravity gradiometer was manufactured based on the concept of differential accelerometry, of which the key element is pairs of high-sensitive capacitive accelerom- 


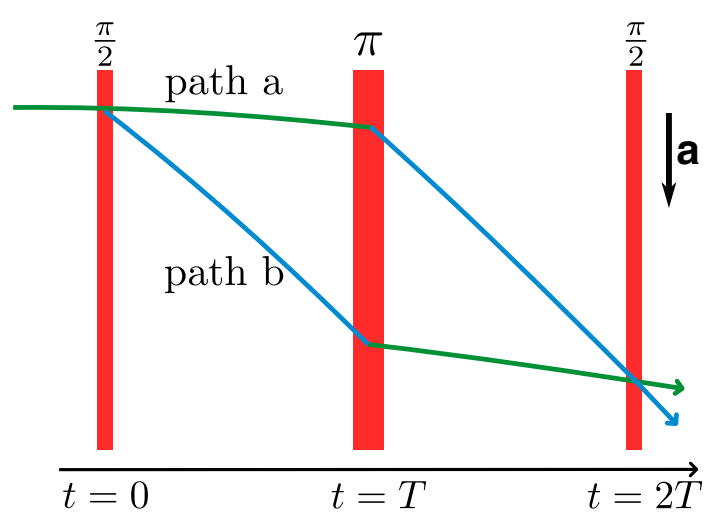

Fig. 1 Scheme of a Mach-Zehnder-type atom interferometer. Clouds atoms are split, reflected and recombined by beam splitter and mirror pulses

eters. The accelerations are obtained through measuring the electrostatic forces that are needed to maintain a test mass at the center of a cage (Rummel et al. 2011). However, this kind of accelerometer suffers from the $1 / f$ noise in the low-frequency part. New kinds of instruments, the atom interferometer-based gravimeter or gradiometer, were developed in various laboratories. These devices utilize cooled atoms as test masses and measure the gravity acceleration or gradients by using the coherence of atomic matter waves. Figure 1 shows the Mach-Zehnder type of an atomic interferometer where laser pulses split, reflect and recombine the atomic wave packets. If the atoms move in free fall, the phase difference between paths $\mathrm{a}$ and $\mathrm{b}$ can be used to extract the gravity information. When two atom interferometers are separated by a distance $D$, the gravity gradient $\gamma$ can be inferred from the differential phase shift $\Delta \Phi$ of atomic matter waves along different paths (Snadden et al. 1998). It reads:

$\Delta \Phi=k\left(a_{2}-a_{1}\right) T^{2}=k \gamma D T^{2}$,

where $k$ is the wave vector for the laser beams, $a_{1}, a_{2}$ are gravity accelerations experienced by the atoms in the two interferometers, $T$ is the free evolution time between successive pulses.

Atom interferometer-based gradiometers, or called as CAI gradiometers, have been demonstrated in various laboratories worldwide, with a good sensitivity at $3 \mathrm{E} / \sqrt{\mathrm{Hz}}$ (Asenbaum et al. 2017). The factors that affect the sensitivity of atomic gradiometers have been systematically analyzed by Sorrentino et al. (2014). A limiting factor is the short interrogation time of atom interferometers, just hundreds of $\mathrm{ms}$ on ground. Since the phase measurement $\Delta \Phi$ is proportional to the square of the interrogation time, this could be much improved in space where the "free fall" condition allows the interrogation time of a few tens of seconds. The "quiet" environment of space can also reduce perturbations, e.g., the seismic effect. Therefore, the CAI gradiometer is expected

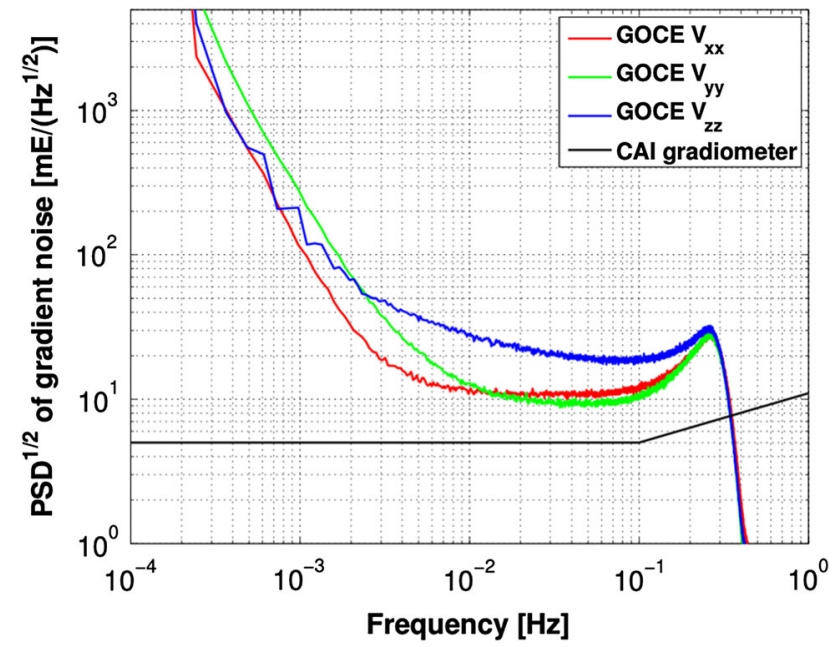

Fig. 2 The root power spectrum density (PSD) of the noise of CAI and GOCE gravity gradients. The performance of the space-based CAI gradiometer refers to Trimeche et al. (2019)

to reach its ultimate performance in space. The sensitivity can potentially achieve the level of a few $\mathrm{mE} / \sqrt{\mathrm{Hz}}$ and even down to $7.0 \times 10^{-2} \mathrm{mE} / \sqrt{\mathrm{Hz}}$ (Rakholia et al. 2017). In addition, the CAI gradiometer shows advantages in its long-term stability. Compared with the GOCE case, it does not show $1 / f$ noise in the low-frequency part, cf. Fig. 2 . The noise power spectrum of CAI gravity gradients is flat down to very low frequencies. This will, on the one hand, contribute to sense more low-frequency gravity signals, and on the other hand, it will simplify the task for whitening the observations that was a challenge in GOCE data processing (Schuh 2003).

The CAI gradiometer has been considered as a future sensor for mapping the Earth's gravity field from space (Yu et al. 2006; Tino et al. 2007; Touboul et al. 2016). A conceptual architecture of a compact CAI gradiometer on board a satellite has been proposed by Carraz et al. (2014). The technical demonstration of this architecture was accomplished by Trimeche et al. (2019), who studied the configuration of the atom interferometers, the requirement on the sensor and its key subsystems, etc. This study shows a three-arm CAI gradiometer in a GOCE-like orbit can measure the three diagonal gravity gradients in the nadir pointing mode. And by applying a precise compensation of orbit rotation, all three gradient components can be obtained at $3.5 \mathrm{mE} / \sqrt{\mathrm{Hz}}$ in Allan deviation. The benefits of such a CAI gradiometer for determining the Earth's gravity field are evaluated by Douch et al. (2018), and there, the pros and cons for different scenarios, i.e., the nadir and inertial Earth's pointing modes, were also discussed. Another project performed a thorough study on a space-based CAI gradiometer for geoscience applications, especially its potential for the detection of some tiny geophysical signals (Migliaccio et al. 2019). At the experimental level, a cloud of cold atoms was successfully created 
on board of a sounding rocket and used for quantum optical sensing (Becker et al. 2018). This was a step further toward the application of atomic sensors in space.

\subsection{Optical clocks}

An optical clock is a device that uses the electron transition frequency in the optical range of the electromagnetic spectrum of atoms as frequency standard. It can achieve an unprecedented accuracy level for the measurement of frequency and time (Ludlow et al. 2015). High-performance optical clocks can probably be used to test the fundamental laws of physics (Delva et al. 2017), redefine time scales (Lodewyck 2019) and improve global positioning and navigation (Schuldt et al. 2018). They can also enable a new measurement concept in geodesy, termed as relativistic geodesy with clocks or chronometric geodesy (Delva et al. 2019). The scheme of this measurement concept is illustrated in Fig. 3. With two clocks operated at different measurement sites and connected by dedicated links, their gravity potential difference $\Delta W$ can be inferred from the fractional frequency difference $\frac{\Delta f}{f}$ (Bjerhammar 1985, 1986). It reads

$$
\frac{\Delta f}{f}=\frac{f_{\mathrm{B}}-f_{\mathrm{A}}}{f_{\mathrm{A}}}=\frac{\Delta W}{c^{2}}+O\left(c^{-4}\right),
$$

where $f_{\mathrm{A}}, f_{\mathrm{B}}$ are the frequencies of an electromagnetic wave measured locally at A and B (the electromagnetic wave transferred from A to B through, for example, an optical fiber), $c$ is the speed of light, and the higher-order term $c^{-4}$ are omitted. If $\mathrm{A}$ is taken as a reference point where $W_{\mathrm{A}}$ is predefined or precisely known, the gravity potential value of point $\mathrm{B}$ can be easily obtained by

$W_{\mathrm{B}}=W_{\mathrm{A}}+\Delta W$

This technique opens the door to directly obtain the gravity potential (difference) value, without having to derive it through measuring its derivatives (gravimetry or gradiometry) or via geodetic levelling plus terrestrial gravimetry as done before.

The performance of optical clocks has been significantly improved since it was first developed in 1990s. Today, optical clocks that are operated in different laboratories worldwide are demonstrated at the uncertainty level of $10^{-18}$ and even beyond. Two independent ytterbium optical lattice clocks (a many-atom system) achieved an uncertainty of $1.4 \times 10^{-18}$ (McGrew et al. 2018). Another type of optical clock (a singleion system) reached even further to $9.4 \times 10^{-19}$ of uncertainty and $1.2 \times 10^{-15} / \sqrt{\tau}$ of instability, where $\tau$ is the averaging time in seconds (Brewer et al. 2019). Efforts are also directed toward a compact clock that can be employed in transportable vehicles and used for measurement campaigns. Such a clock was built in a car trailer and reported with an uncertainty of $7.4 \times 10^{-17}$ (Koller et al. 2017), and its demonstration for field measurement has been made by Grotti et al. (2018).

To enable the ultra-precise comparison of clocks over long distances, frequency transfer techniques that use optical fibers, microwaves, free-air laser beams and the timesynchronization method through navigation satellites are extensively studied and developed (Petit et al. 2014; Riehle 2017). Among them, the frequency link via optical fibers reached a fractional uncertainty of $2.5 \times 10^{-19}$ over 1400 $\mathrm{km}$, which meets the need for the comparison of $10^{-18}$ and even more accurate clocks (Lisdat et al. 2016). Two-way optical laser links were also experimented. They can potentially achieve a free-air frequency transfer with an uncertainty of $10^{-18}$ (Bergeron et al. 2019; Sinclair et al. 2019). Alternatively, the microwave link is considered as an efficient and economic method. It will be used for the comparison between atomic clocks in space and on ground (Delva et al. 2012) and might achieve an uncertainty of $10^{-16}$ (Laurent et al. 2015). A large-distance link can also be realized using the time-synchronization technique via navigation satellites (Leute et al. 2016; Hachisu et al. 2014). It achieves $10^{-16}$ at several days of averaging time (Petit et al. 2017). These techniques will keep improving to support the link between distant clocks in various scenarios.

Optical clocks are opening up a new field in geodesy. They are becoming comparable with classic geodetic methods to achieve the centimeter/decimeter level in inferring height information over long distances (Takano et al. 2016; Lisdat et al. 2016). This makes optical clocks applicable for the unification of local height systems (Wu et al. 2018) or realizing a consistent global height reference system (Mehlstäubler et al. 2018). They are also highly relevant for various geodetic and geophysical applications, such as the realization of a space-time reference system (Berceau et al. 2016), monitoring vertical surface motions (Bondarescu et al. 2015) and the determination of the Earth's gravity field (Müller et al. 2018). Using clocks for determining a local geopotential model with a high spatial resolution was quantitatively investigated by Lion et al. (2017). Moreover, space-borne optical clocks were proposed for physical and geodetic applications (Gill et al. 2008). Efforts toward a compact, high-performance and ultra-stable clock that is aimed to be employed in space were made by Origlia et al. (2018). We will follow the idea proposed by Müller et al. (2018) to evaluate the benefits of high-performance clocks for mapping the Earth's gravity field from space. 
Fig. 3 Scheme for clock-based geodesy. The comparison of clocks' frequencies can be used to infer the gravity potential difference between two points

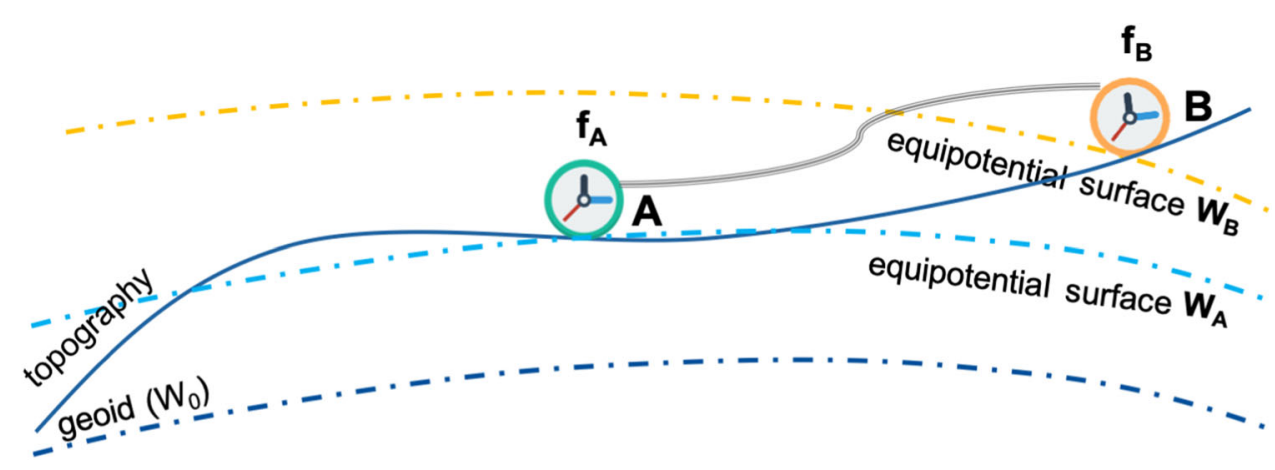

\section{Closed-loop simulations}

\subsection{Flowchart of simulation}

Numerical simulations are performed to quantify the benefits of the CAI gradiometer and optical clocks for the determination of Earth's gravity field. We designed the simulation in the time domain. It is a closed-loop procedure and is targeted to fully map the sensor sensitivity in terms of gravity field coefficients. A general overview of the simulation workflow is shown in Fig. 4. The noisy observations that comprise a signal and a noise part are the main input for gravity field recovery. The signals for each type of observations (potential and gradients) are spherical harmonics synthesized from a reference gravity field model along a predefined satellite orbit. Meanwhile, the noise is generated based on the sensors' characteristics. Then, normal equations are established from the noisy observations and a least-squares adjustment was applied to estimate the gravity field coefficients as well as their standard deviations. Finally, the recovered models are compared with the input reference model and their differences are taken for the evaluation.

We took the EIGEN-6c4 as reference gravity field model. It is an accurate, high-degree solution that combines the contribution of satellite and terrestrial gravimetry observations (Förste et al. 2014). The reference model was truncated at degree and order (d/o) 350. The coefficients above are omitted, mainly for reducing the computational time. Through tests, we could verify that the omission part has nearly no effects on the recovered solutions up to d/o 240 .

We took a GOCE orbit in most simulation cases for the sake of having an intuitional comparison of gradiometrybased solutions. A 71-day orbit that starts from March 2, 2013, was chosen. During this period, the satellite orbit was at a mean altitude of about $240 \mathrm{~km}$, a repeat cycle of 65 days and an inclination of $96.5^{\circ}$. Since the orbit is only used for geolocating observations, we used the reduced-dynamic orbit for our simulation. It is a continuous, smooth and accurate solution, with a position error of $1-2 \mathrm{~cm}$ (Bock et al. 2014). The original orbit was given at an interval of $10 \mathrm{~s}$, and it was interpolated according to our needs by using the spline method. A GRACE orbit was also used, in the case to investigate the potential of clocks for detecting time-variable gravity signals. Observations along a 1-month GRACE orbit were simulated and then used for deriving monthly gravity field solutions.

According to the preliminary design of a space-based CAI gradiometer, it does not suffer from $1 / f$ noise in the lowfrequency part and can achieve a sensitivity of $3.5 \mathrm{mE} / \sqrt{\mathrm{Hz}}$ in Allan deviation, which corresponds to a white noise with a one-sided amplitude spectral density (ASD) of $3.5 \times \sqrt{2} \approx$ $5.0 \mathrm{mE} / \sqrt{\mathrm{Hz}}$ (Trimeche et al. 2019; Douch et al. 2018). We therefore simply assumed a white noise of $5.0 \mathrm{mE} / \sqrt{\mathrm{Hz}}$ for all CAI gradients, see also Fig. 2. It is hard to specify the noise of clock measurements in space, since the knowledge of space-based optical clocks is still very poor. However, as discussed in Sect. 2.2, the best clocks in laboratories on ground can achieve a performance of $10^{-18}$ in terms of fractional frequency. The laser link is also promising to realize the frequency transfer at the level of $10^{-18}$ in free space. We therefore assumed that the future clock measurements can potentially achieve a desired level of $1.0 \times 10^{-18}$, including uncertainties from both the clocks and links. In addition, the clock measurements are assumed with a white-noise behavior in the relevant frequency range.

\subsection{Earth's gravity field}

The Earth's global gravity field is commonly expressed in a spherical harmonic expansion, with the gravity potential $V$ of any point on the Earth's surface described as (HofmannWellenhof and Moritz 2006)

$$
\begin{aligned}
V(r, \theta, \lambda)= & \frac{G M}{R} \sum_{n=0}^{\infty}\left(\frac{R}{r}\right)^{n+1} \sum_{m=0}^{n}\left[\bar{C}_{n m} \cos (m \lambda)\right. \\
& \left.+\bar{S}_{n m} \sin (m \lambda)\right] \bar{P}_{n m}(\cos \theta),
\end{aligned}
$$

where $G M$ is the gravitational constant of the Earth, $R$ the equatorial radius of the reference ellipsoid, $r, \theta, \lambda$ are the spherical coordinates of the point, $n, m$ are the spherical harmonic degree and order, $\bar{P}_{n m}$ are the fully normalized 
Fig. 4 The flowchart of the closed-loop simulation

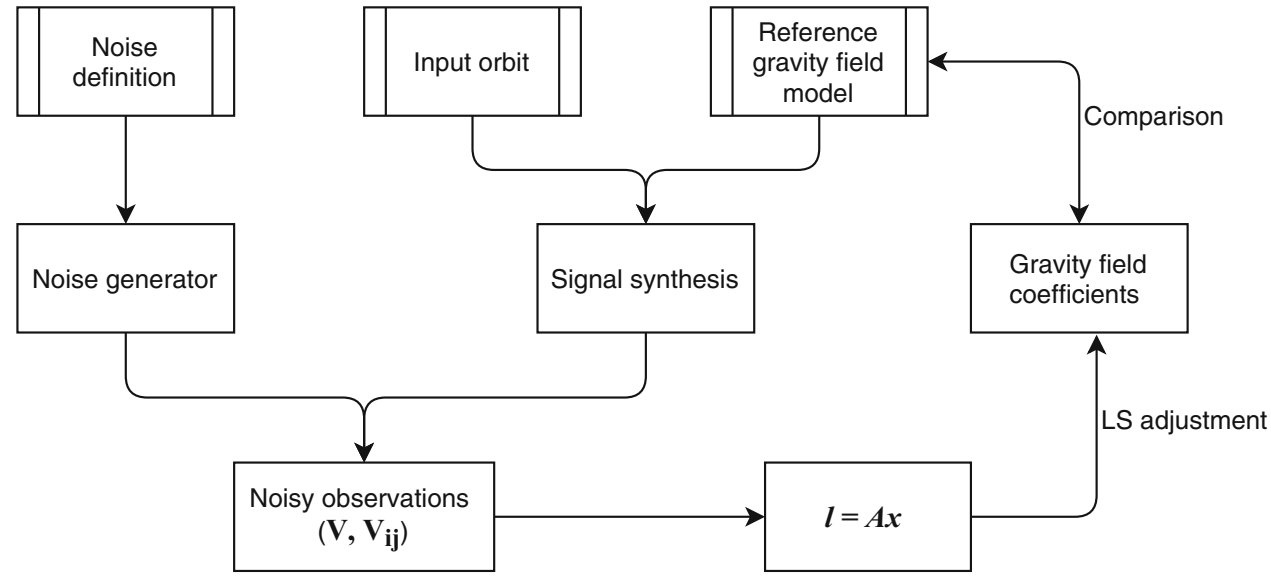

associated Legendre functions, and $\bar{C}_{n m}, \bar{S}_{n m}$ are the spherical harmonic coefficients that are the unknown parameters to be estimated for the gravity field recovery. Gravity gradients are point-wise measurements of the second-order partial derivatives of gravity potential. They can be written as

$V_{i j}=\frac{\partial^{2} V}{\partial x_{i} \partial x_{j}}$,

where $V_{i j}$ are different gradient components, and the subscripts $i, j$ denote one of the coordinate axes (e.g., $r, \theta, \lambda$ in a spherical coordinate system, and $x, y, z$ for a Cartesian system). The gravity gradients are practically measured in the instrument frame, like the gradiometer reference frame (GRF), while gravity gradients are conveniently modelled in the so-called local north-oriented frame (LNOF, see GOCE standards (ESA 2014)). We need to unify the measurements and the model to the same reference frame that is written as:

$V_{i j}^{F_{2}}=R\left(\frac{\partial^{2} V}{\partial x_{i} \partial x_{j}}\right)^{F_{1}} R^{T}$,

where $F_{1}, F_{2}$ denote two different reference frames, and $R$ represents the rotation matrix between both frames. This equation will be used to set up the functional model of the gravity gradients for gravity field recovery.

A rigorous least-squares adjustment was applied to estimate the gravity field coefficients. We first needed to assemble the normal equation systems for each type of observations. In order to optimize the computational task, the normal equations were assembled arc-wise, which on the one hand reduces the size of the design matrix to save memory space and on the other hand makes the procedure easy for parallel computation. For the processing of real GOCE gravity gradients, we constructed the stochastic model from the post-fit residuals of each arc, in which empirical parameters were also introduced ( Wu 2016). But this is not the case for this work. A unit matrix is taken as stochastic model because the noise is treated as white. After the normal equation systems were assembled, a direct inversion method was applied to estimate the gravity field coefficients as well as their standard deviations (Koch 1999). Moreover, a joint solution was recovered in order to combine the contributions of CAI gradiometry and clock observations. Their individual normal equation systems were combined with proper weighting factors, which were taken as the reciprocal of the a posteriori variance of unit weight for each solution. In the end, the recovered models were compared with the input reference model, and their differences are used to retrieve the true model errors. We also obtained formal errors of the solution in the least-squares adjustment procedure that are standard deviations of the estimated coefficients. The true errors and formal errors are finally used for the model evaluation.

\section{Results and discussion}

\subsection{Solutions for CAI gradiometry}

We followed the conceptual design of a space-based CAI gradiometry mission that was accomplished by Trimeche et al. (2019). CAI gradiometry is proposed to be realized in the nadir pointing mode, which corresponds to the usually Earth-pointing satellite attitude that the y-axis (the crosstrack direction) rotates together with the satellite frame at the rate of $\Omega_{\text {orb }}$ to keep the z-axis always pointing to the Earth's center. $\Omega_{\text {orb }}$ is the orbit rotating rate and is about $1.16 \mathrm{mrad} / \mathrm{s}$ for a low-satellite orbit with a revolution period of about $5400 \mathrm{~s}$. In this pointing mode, a one-arm gradiometer can be realized to precisely measure the cross-track gradient $V_{y y}$, while only a three-arm gradiometer can simultaneously measure the three diagonal gradients $V_{x x}, V_{y y}$ and $V_{z z}$. However, $V_{x x}$ and $V_{z z}$ cannot be measured as precisely as $V_{y y}$ without dedicated procedures due to a centrifugal force error caused by the orbit rotation. A compensation scheme was proposed to counteract the orbit rotation by tilting the Raman mirror 


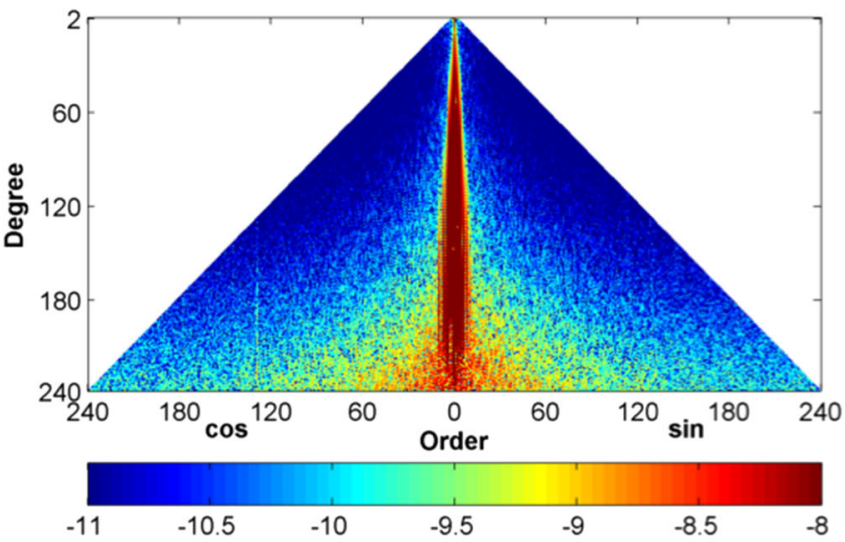

(a) True errors

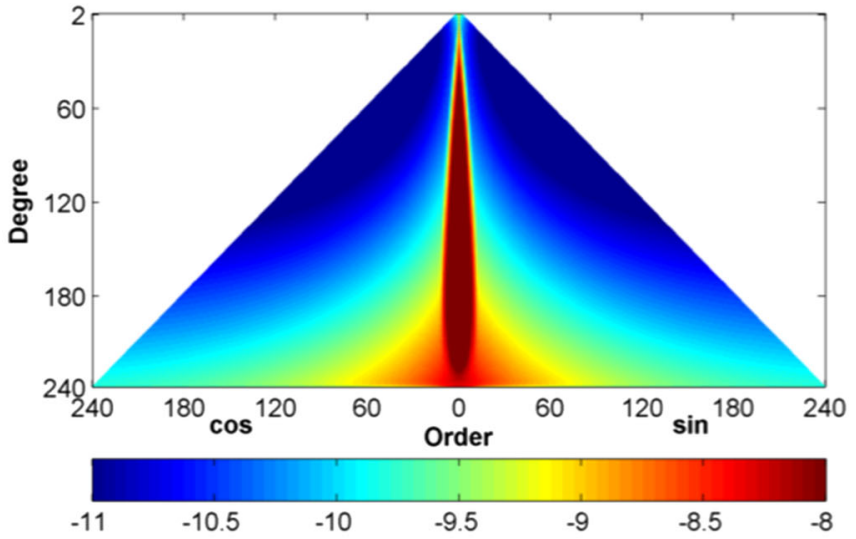

(b) Formal errors

Fig. 5 True errors and formal errors for the gravity field solution of one-arm CAI gradiometry. Both are displayed in logarithmic scale

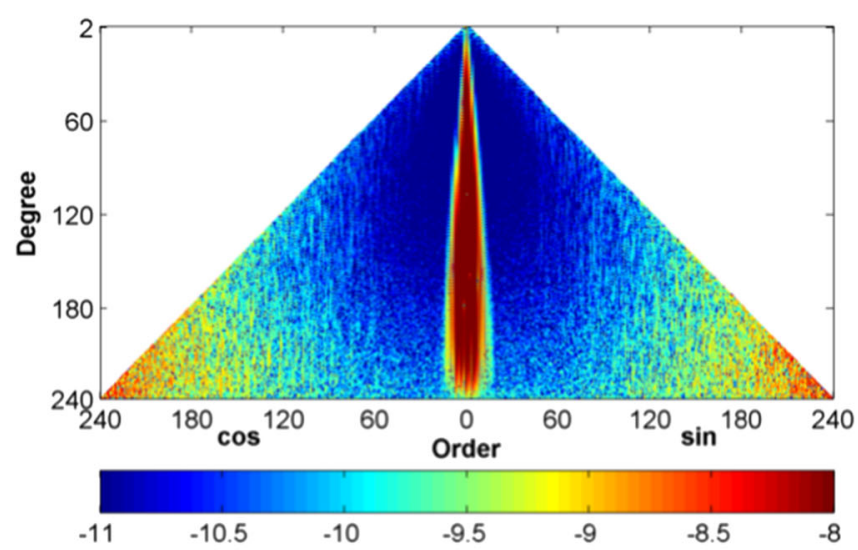

(a) $V_{x x}$

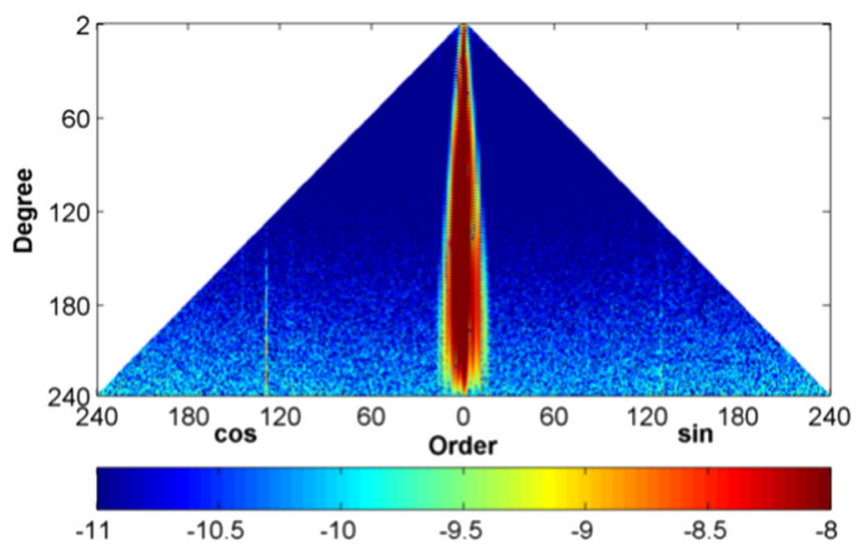

(b) $V_{z z}$

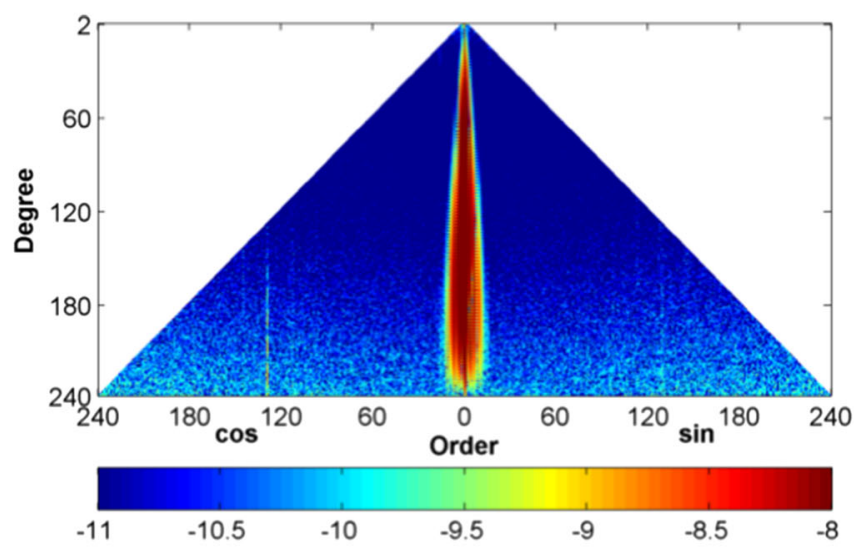

(c) Combination of $V_{x x}, V_{y y}, V_{z z}$

Fig. 6 True errors of the gravity field solutions for three-arm CAI gradiometry, in logarithmic scale

of the atomic interferometer. The technical demonstration of this scheme is discussed by Trimeche et al. (2019). When the rotation compensation is precisely applied, all the three diagonal gradients can be obtained with the same accuracy.
We evaluated the performance of the one- and three-arm CAI gradiometry for determining the Earth's gravity field. Gravity gradients were simulated along the selected GOCE orbit, as described in Sect. 4. Their noise was assumed at the same level of $5.0 \mathrm{mE} / \sqrt{\mathrm{Hz}}$. The orbit was resampled at 
an interval of $2 \mathrm{~s}$, which is technically achievable for CAI gradiometry. With such an interval, a reasonable amount of observations can be obtained for gravity field modelling. We then recovered component-wise gravity field solutions for all gradients. A joint solution was recovered as well for the three-arm gradiometry case that combines the contribution of the three diagonal gradient components. All solutions were recovered up to d/o 240 .

The one-arm gradiometry solution is presented in Fig. 5. The true errors and formal errors are displayed in logarithmic scale. Both are consistent and show a homogeneous pattern. And they indicate that the one-arm gradiometer has natural deficits for determining the Earth's gravity field, as it is mainly sensitive to the sectorial and near-sectorial gravity field coefficients, of which the order is equal or close to the degree. It means that the one-arm gradiometer is capable to capture only the structure of the gravity field along the longitude direction when it were on board of a near-polar satellite in the nadir pointing mode. Figure 6 shows the solutions of the three-arm gradiometry. Only the true errors are shown there as the formal errors show a similar behavior. The solution of $V_{y y}$ is not shown because it senses the same signal in both cases. Except the zonal and near-zonal coefficients degraded by the polar gaps of the GOCE orbit, a complete gravity field solution can be retrieved from three-arm gradiometry. The challenge lies in its technical realization, with one key point to implement compensation systems for the rotation of the satellite.

Figure 7 shows the degree medians of the true errors. It confirms that $V_{y y}$ solutions are identical for the one-arm and three-arm gradiometry. The solution from $V_{x x}$ is generally close to that of $V_{y y}$ as their signal-to-noise ratios are at the same level. But above about d/o 50, the $V_{x x}$ solution is slightly poorer. This is related to the inhomogeneous sensitivity of the along- and cross-track gradients that $V_{x x}$ is more sensitive to the zonal and near-zonal coefficients, while $V_{y y}$ is more sensitive to the sectorial parts, as shown in Figs. 5a and 6a. The solution from $V_{z z}$ shows the best performance, about twice better than the other components above $\mathrm{d} / \mathrm{o} 50$. This is attributed to its bigger signal-to-noise ratio that the signal is about twice of that of the other components, while the noise is assumed at the same level, and as well as its more homogeneous error characteristics. Consequently, $V_{z z}$ contributes most to the combined solution. For all solutions, the errors increase quickly below $\mathrm{d} / \mathrm{o} 40$. Considering that the CAI gradients have flat noise down to a very low-frequency part, this manifests that gradiometry is conceptually less sensitive to the low-degree gravity signals. There, other kinds of gravity observations are required, e.g., potential values obtained from clocks.

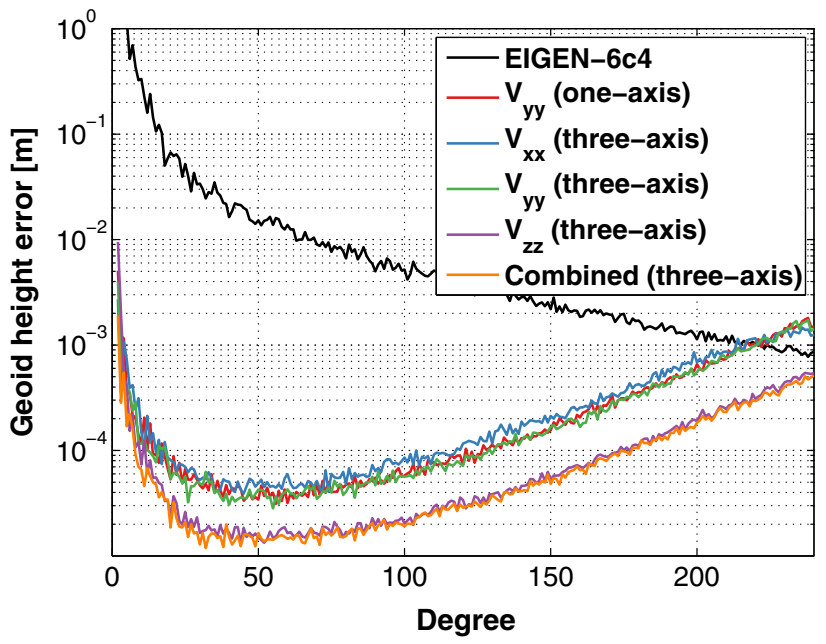

Fig. 7 Degree medians of the true errors for the recovered CAI gradiometry solutions, in terms of geoid height

\subsection{Solutions for clocks}

A dataset with a whole coverage over the world is required to recover the global gravity field. We assumed a hybrid clock network that contains a number of reference clocks on ground and a target clock on board a low-orbit satellite to obtain such a clock data set. The clocks on ground and in space can be compared with direct laser links. They can also be linked via some relay satellites in high orbits, like a geostationary orbit. The transponders/clocks in geostationary orbit receive the signals from reference clocks on ground and retransmit them to the target clock on the LEO satellite. With the direct and indirect links, the predefined or known gravity potential values of the reference clocks can be transmitted to the space-based clock. As a further concept, we may also follow the GRACE-like mission scenario by using two clocks on board of twin co-orbiting satellites to measure the potential difference between them. This scenario can get rid of the dependence on reference clocks on ground. These results are not discussed here. In this paper, we assumed that a global dataset of gravity potential values is obtained from a hybrid clock network proposed in the first scenario.

Three datasets of gravity potential values were simulated along the same orbit as that used for the CAI simulation. One is the clock measurements with the desired level of $1.0 \times 10^{-18}$, including frequency uncertainties from both the clocks and links. Another two represent an optimistic and a pessimistic case with respect to the desired one. They have the noise levels of $1.0 \times 10^{-19}$ and $1.0 \times 10^{-17}$, respectively. Perturbations that are related to the tides, non-gravitational forces, etc., are assumed to be precisely modelled or measured. In addition, the second-order Doppler effect that propagates the error of the satellite velocity $v$ to the frequency measurement as $\frac{v \cdot \delta v}{c^{2}}$ has been neglected. In case to reduce its 


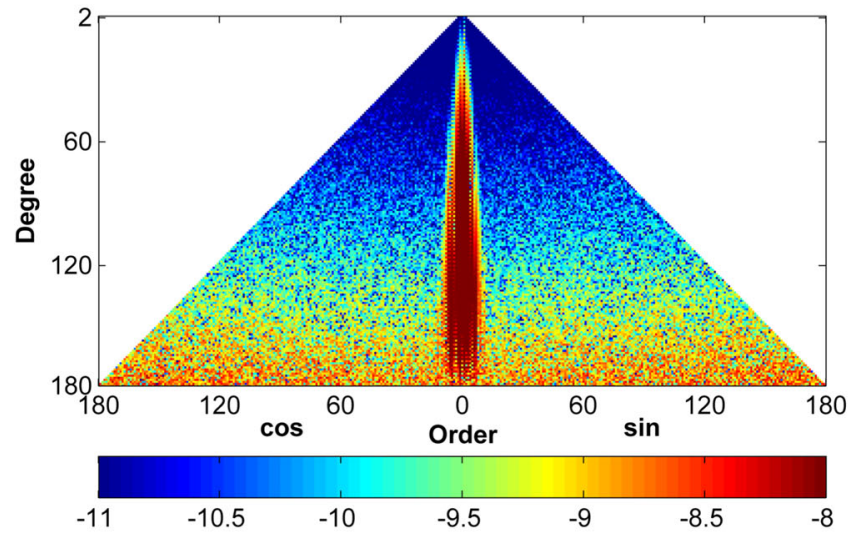

(a) True errors

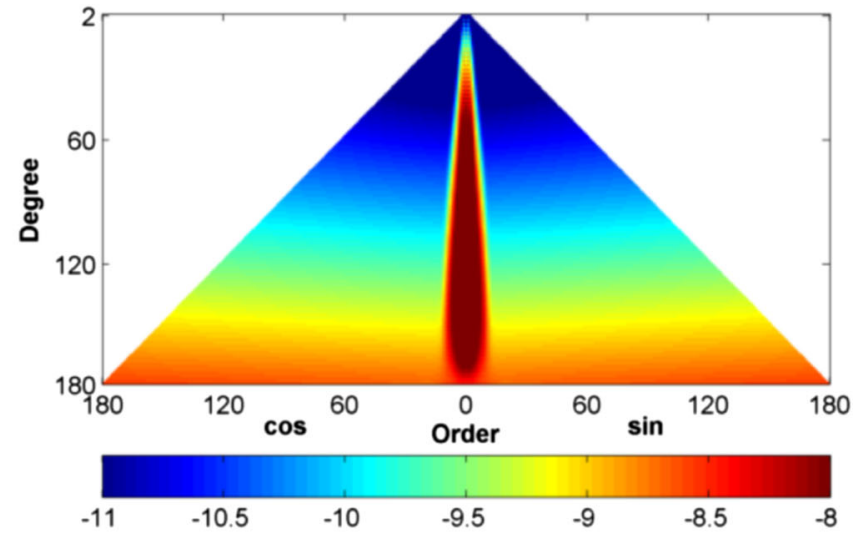

(b) Formal errors

Fig. 8 True and formal errors of the gravity field solution for clocks with an uncertainty level of $1.0 \times 10^{-18}$, in logarithmic scale

contribution down to the level of $10^{-18}$ in terms of frequency, the satellite velocity has to be determined with an accuracy level of $10^{-5} \mathrm{~m} / \mathrm{s}$. Here, we resampled the original orbit with an interval of $5 \mathrm{~s}$ in order to obtain a reasonable number of observations for gravity field recovery. The assumption of this sampling rate, however, is an ideal case when the stability of clocks is taken into account. More discussion on this topic will be addressed later.

Clock solutions are recovered up to degree and order 180. The true and formal errors for the $10^{-18}$ clock solution are displayed in Fig. 8. It shows that clocks have a homogeneous sensitivity to all order coefficients at the same degree. The clock solution has the best performance in the low-degree part, while the errors gradually increase with degree. This indicates that clocks are most sensitive to the large-scale or long-wavelength gravity field signals. It makes clock-based gravimetry complementary to gradiometry or other kinds of gravimetry. The same conclusion can be drawn from the degree medians of the true model errors, as shown in Fig. 9. In addition, the solution from clocks with an uncertainty of $1.0 \times 10^{-18}$ is about one order of magnitude better than that from $1.0 \times 10^{-17}$ clocks for all degrees, and about one order of magnitude worse than that from $1.0 \times 10^{-19}$ clocks. The degree errors of the recovered gravity field models are consistent with the assumed noise levels.

Since clocks are sensitive to the low-degree gravity field coefficients, we further investigated their potential for detecting time-variable gravity signals. In order to have a direct comparison with the temporal gravity field that is represented by the GRACE monthly solutions, we simulated clock observations along a 1-month GRACE orbit with an interval of 5 s. The gravity field solutions are recovered up to d/o 60 .

To evaluate the recovered models, we compared the degree variances of their true errors with the GRACE-based timevariable signals as well as the corresponding cumulative values. The results are shown in Fig. 10. Clocks with an

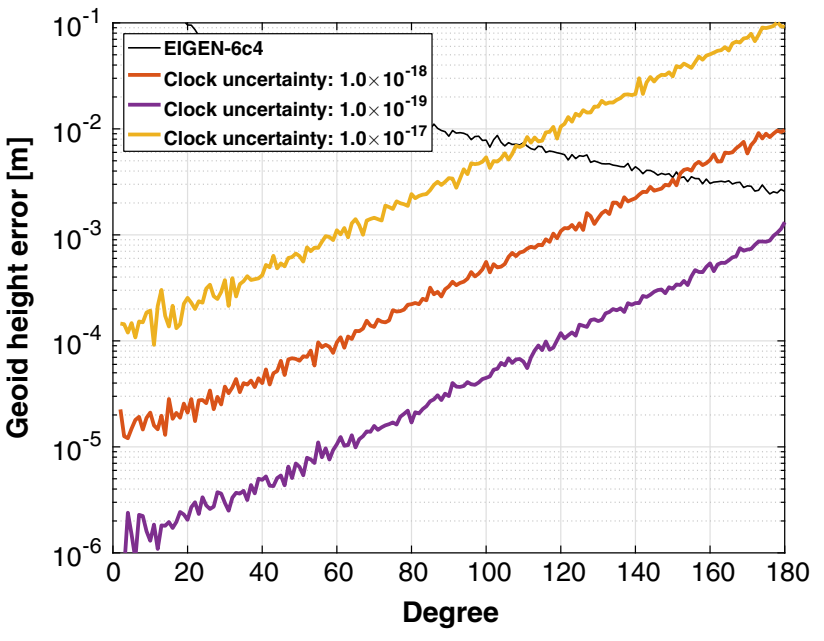

Fig. 9 Degree medians of the true model errors. Comparison of the gravity field solutions that are recovered from clock measurements with different magnitudes of uncertainties

uncertainty of $10^{-18}$ are capable to retrieve the time-variable signals below d/o 12 . The contribution can go up to degree 30 for $10^{-19}$ clocks, while it is only possible to sense signals of degree 2 for clocks at the level of $10^{-17}$. If the cumulative values are accounted, clocks with uncertainties of $10^{-17}, 10^{-18}, 10^{-19}$ have the potential to detect the timevariable signals to degrees 5, 25 and 60 , respectively.

From the perspective of gravity field recovery, it is desirable that a large amount of observations can be acquired to improve the parameter estimation accuracy. For that, above we assumed the clock measurements with a sampling interval of $5 \mathrm{~s}$. This is however an ideal hypothesis when we take the clock's stability into account. Today, as the best case, two independent optical clocks can reach the $4.8 \times 10^{-17}$ stability in $1 \mathrm{~s}$ (Oelker et al. 2019). Other clocks are reported at the instability level of $10^{-16} / \sqrt{\tau}$, where $\tau$ is the averaging 


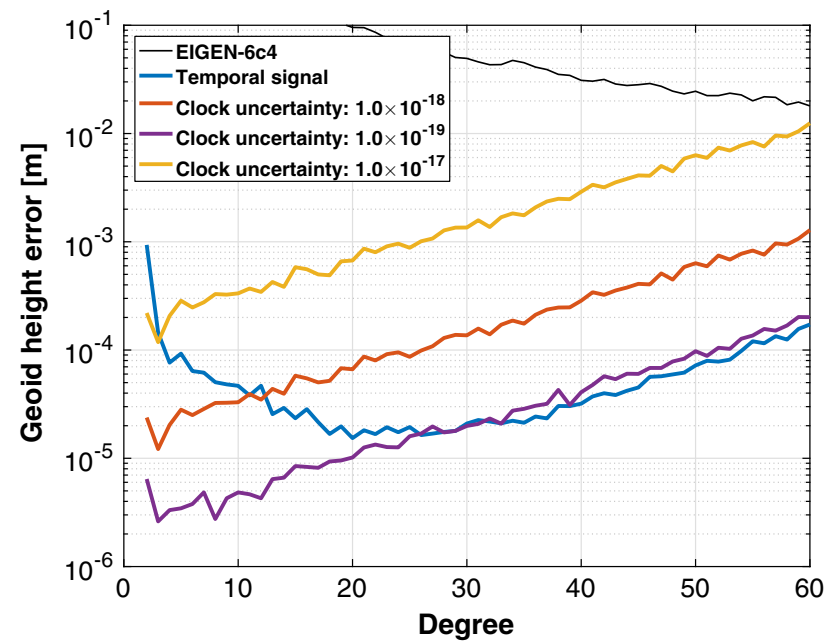

(a) Degree variances

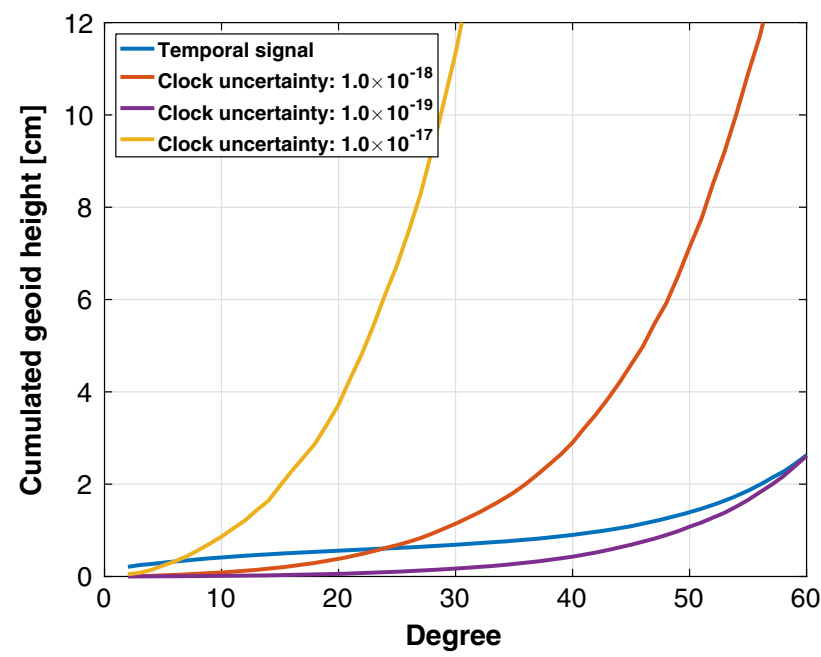

(b) Cumulative errors

Fig. 10 Comparison of the monthly clock solutions with time-variable gravity signals represented by the GRACE solution

time in seconds. Taking both levels as examples, we suppose to get clock measurements with uncertainties of $4.8 \times 10^{-18}$ and $1.0 \times 10^{-17}$ every $100 \mathrm{~s}$. Clock observations with such uncertainties were simulated along the 71-day GOCE orbit, at a mean altitude of $240 \mathrm{~km}$. For the purpose of comparison, here we also included a 71-day GRACE orbit starting from January 1, 2006, at a mean altitude of around $475 \mathrm{~km}$. For each case, we then have 61,344 observations to estimate the gravity field up to $\mathrm{d} / \mathrm{o} 60$.

The degree medians of the true model errors are shown in Fig. 11. When the GOCE orbit was used, the clock with an uncertainty of $4.8 \times 10^{-18}$ at 100 s results in the best solution, which is about one time better than that from the other clock. The comparison between the orange and purple curves indicates that the orbit has an evident impact on the recovered models. When identical clocks are employed at different

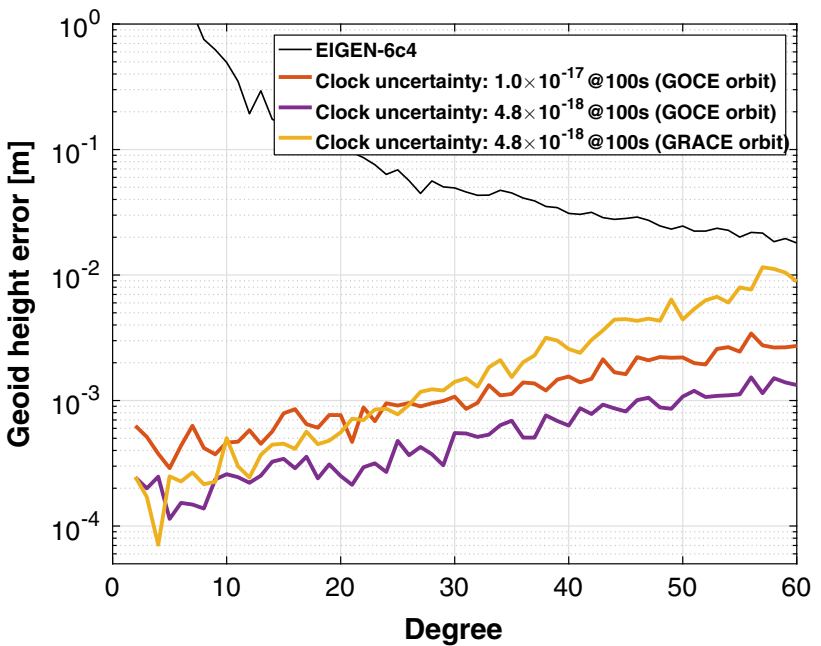

Fig. 11 Degree medians of the true errors for the models recovered from clocks with different stabilities. One is assumed as $1.0 \times 10^{-16} / \sqrt{\tau}$ and the other one is $4.8 \times 10^{-17}$ at $1 \mathrm{~s}$, which allow to get the clock measurements with uncertainties of $1.0 \times 10^{-17}$ and $4.8 \times 10^{-18}$ every $100 \mathrm{~s}$. In addition, clocks at different satellite's altitudes (GOCE and GRACE) are compared

altitudes ( $240 \mathrm{~km}$ vs. $475 \mathrm{~km}$ ), they show a comparable sensitivity to the very low-degree gravity field coefficients (say below d/o 12). However, the sensitivity of the clock to the higher harmonics of the gravity field decreases quickly at the higher altitude. At degree 60, the model error is nearly one order of magnitude higher than that of the lower orbit. This confirms that the Earth's gravity potential attenuates quickly with the altitude, so that other types of gravimetric measurements like gravity gradiometry are necessary to determine the medium- and short-wavelength gravity field parts. The red and orange curves intersect around degree 25 . This manifests that a lower orbit is more significant than the sensitivity of the clock to recover the gravity field coefficients above degree 25 . For clocks with the assumed instabilities, just one of them in space does not seem to being able to collect a comparable number of observations as other satellite missions in a short time period. The long averaging time to achieve a desired uncertainty level makes it a challenge to use clocks for retrieving global time-variable gravity signals.

In order to obtain observations as many as possible, in principle, one should have more clocks in space or a mission with a longer time span. This can help to relax the requirement on the stability. Prospectively, the rapid development of quantum technology could bring a further breakthrough for the clock's stability. As an example, the Thorium clock, a kind of nuclear clock that uses the frequency of a nuclear transition as its reference frequency, can potentially achieve an advanced level of uncertainty and stability (Seiferle et al. 2019). Besides, one can consider using clocks in space to determine only the very low-degree coefficients, e.g., the degree- 2 coefficients or the so-called J2 perturbations. 


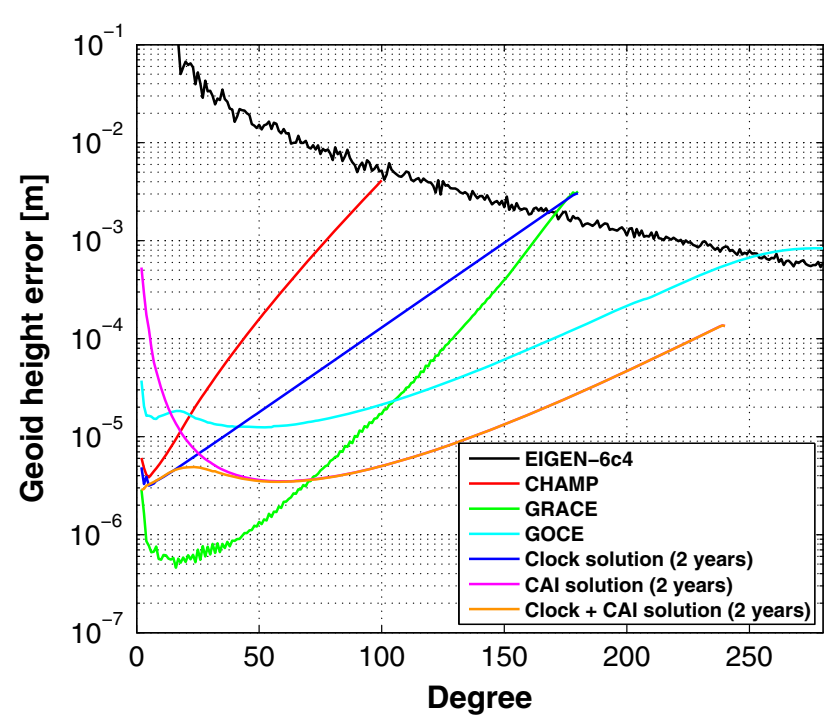

Fig. 12 Degree medians of the formal errors, in terms of geoid height. To compare with the CHAMP (AIUB-CHAMP03s), GRACE (ITGGrace2010s) and GOCE (EGM_TIM_RL5) solutions, we scaled our CAI, clock and their combined solutions to 2 years

\subsection{Solution for the combined case}

To illustrate the strength of each type of observation, the clock data at the level of $1.0 \times 10^{-18}$ and the three-arm CAI gravity gradients are jointly analyzed to derive a combined solution. In order to have an intuitive impression on the quality of the combined model for these novel sensors, we compared it with other satellite-only gravity field solutions like GRACE and GOCE. We would like to point out here that it is challenging to perform a strict comparison between the simulated solutions with the realistic models, because the performance of the gravity field models is affected by a number of factors, like the volume of data, the type of observations, the constrain conditions, the satellite altitude and so on. The GRACE- and GOCE-like satellite-only gravity field models were recovered from multiple years of observations. For the sake of a (relatively) reasonable comparison, we scaled our 71-day models to adapt to a longer mission lifetime, e.g., 2 years, and to a high-sampling rate like $1 \mathrm{~s}$. The model errors were extrapolated by applying the $1 / \sqrt{t}$ rule, where $t=N_{1} / N_{2}$ and $N_{1}, N_{2}$ are the number of observations for datasets 1 and 2 . This is valid on the condition that the observation noise is purely stochastic, but might be optimistic for the practical case as realistic noise is more likely to be non-stationary over time.

The comparison of our scaled 2-year solutions with the other satellite-only gravity field models is shown in Fig. 12. In order to get rid of the systematic errors of the realistic models, we took the formal errors for our analysis. And to remove the polar gap effect that is related to the GOCE orbit, the degree medians were compared. The CAI gradiometry solution is about $2-5$ times better than the GOCE solution starting from $\mathrm{d} / \mathrm{o}$ around 50 . This attributes to the lower noise level of CAI gradients. Some improvement is also visible below $\mathrm{d} / \mathrm{o} 50$, because of the flat noise of the CAI gravity gradients down to low frequencies. The clock solution shows a better performance than the CHAMP and GOCE solutions in the low-degree part, especially below around d/o 20. In this frequency domain, the CHAMP and GOCE solutions are mainly computed from the satellite-to-satellite high-low (SST-hl) tracking data. That manifests that clocks onboard satellites might be an alternative way to SST-hl for determining the low-degree coefficients. Generally, GRACE shows the best performance below about degree 70 thanks to its range-rate observations. This kind of measurements can now also be obtained by a laser ranging system in GRACE-FO, which might result in an even better gravity field solution.

\section{Conclusions and future perspectives}

The progress in quantum sensor technology provides new measurement concepts for future gravity missions. Optical clocks on board of a satellite can be used to obtain the gravitational potential values along the orbit through frequency comparison with ground clocks. As the gravitational potential is a scalar quantity, the transformation between different reference frames is not necessary for clock observations. It can thus get rid of, for example, attitude errors and saves much efforts in data processing. Clock measurements are sensitive most to the low-degree coefficients. Their sensitivity to high-frequency gravity signals gradually decreases with degree. Clocks are possible to detect the large-scale temporal gravity field. Clocks with an uncertainty level of $1.0 \times 10^{-18}$ are capable to retrieve temporal gravity signals below d/o 12 , while $1.0 \times 10^{-17}$ clocks might only be useful to detect the signals of degree 2 . But this poses a challenge for clocks to achieve such uncertainties in a short time, say $5 \mathrm{~s}$, which is not achievable with today's technology, yet. Another promising sensor is the CAI gradiometer which uses pairs of atom interferometers to measure gravity gradients. It can potentially achieve a high sensitivity $(5.0 \mathrm{mE} / \sqrt{\mathrm{Hz}})$ and long-term stability (flat noise down to very low frequencies). The technical design demonstrates the feasibility to realize a three-arm CAI gradiometer in the nadir pointing mode by applying compensation to counteract the orbit rotation. With such a design, the three main diagonal gravity gradients can be measured with the same high accuracy. These high-accurate CAI gradients lead to a better solution than GOCE by a factor of 2-5 above $\mathrm{d} / \mathrm{o}$ 50. Benefits are also visible in the lower-degree part because of the flat-noise behavior of the CAI gravity gradients. But the improvement is not so prominent as that above $\mathrm{d} / \mathrm{o} 50$. In the frequency range between the clock and 
CAI gradiometer contributions, the best gravity solution is obtained from GRACE-like range-rate observations.

Optical clocks and the CAI gradiometer are emerging sensors for geodetic measurements. The application of them in space to determine the global gravity field still requires further efforts. The procedure for the comparison between clocks in space and on ground has to be elaborated, where the Doppler effect which is dependent on the precision of satellite's velocity should be seriously handled. Also, the configuration of the clock network, which may include a number of ground clocks, a few relay satellites and a space-based clock, needs to be optimized to obtain a global distribution dataset. Then, attention should be paid on identifying an elaborated noise behavior of the clocks. As for CAI technology, it can not only be used for measuring of gravity gradients, but also appear to support classical electrostatic accelerometry to measure the non-gravitational forces as, for example, needed in GRACE-type missions. The long-term stability of CAI technology might also help to simplify the calibration procedure and reduce systematic errors in the observations. In addition, both novel sensors may be considered for detecting temporal gravity field signals. But then aliasing errors caused by the imperfect background models, e.g., atmospheric and ocean models, have to be taken into account.

Acknowledgements Open Access funding provided by Projekt DEAL. This research was funded by the Deutsche Forschungsgemeinschaft (DFG, German Research Foundation) under Germany's Excellence Strategy -EXC-2123/1 (Project-ID: 390837967) and the Collaborative Research Center SFB 1128 "Relativistic Geodesy and Gravimetry with Quantum Sensors (geo-Q)." This work was also partly supported by the European Space Agency (ESA) project "Study of a CAI gradiometer sensor and mission concepts." We gratefully acknowledge the International Space Science Institute (ISSI) for providing support to participate at international workshops on the topic of "Spacetime metrology, clocks and relativistic geodesy."

Author contributions JM and HW conceptualized and designed the research and analyzed the results. HW prepared the data input, performed the computation and wrote the manuscript. JM provided critical comments to the manuscript and approved the final version.

Data availability The reference gravity field models that were used in this study are available in the International Centre for Global Earth Models (http://icgem.gfz-potsdam.de/tom_longtime). The GOCE data were provided by the European Space Agency (ESA) via the virtual online archive (https://earth.esa.int/web/guest/-/goce-data-access-7219). The GRACE data were obtained from the Jet Propulsion Laboratory (JPL)'s PO.DAAC Drive (ftp://podaac.jpl.nasa.gov/allData/grace/L1B/JPL/). Other datasets generated in this study are available from the corresponding author on request.

Open Access This article is licensed under a Creative Commons Attribution 4.0 International License, which permits use, sharing, adaptation, distribution and reproduction in any medium or format, as long as you give appropriate credit to the original author(s) and the source, provide a link to the Creative Commons licence, and indicate if changes were made. The images or other third party material in this article are included in the article's Creative Commons licence, unless indicated otherwise in a credit line to the material. If material is not included in the article's Creative Commons licence and your intended use is not permitted by statutory regulation or exceeds the permitted use, you will need to obtain permission directly from the copyright holder. To view a copy of this licence, visit http://creativecomm ons.org/licenses/by/4.0/.

\section{References}

Asenbaum P, Overstreet C, Kovachy T, Brown DD, Hogan JM, Kasevich MA (2017) Phase shift in an atom interferometer due to spacetime curvature across its wave function. Phys Rev Lett 118:183602. https://doi.org/10.1103/PhysRevLett.118.183602

Barrett B, Bertoldi A, Bouyer P (2016) Inertial quantum sensors using light and matter. Phys Scr 91(5):053006. https://doi.org/10.1088/ 0031-8949/91/5/053006

Becker S, Brockmann JM, Schuh WD (2014) Mean dynamic topography estimates purely based on GOCE gravity field models and altimetry. Geophys Res Lett 41(6):2063-2069. https://doi.org/10. 1002/2014GL059510

Becker D, Lachmann MD, Seidel ST, Ahlers H, Dinkelaker AN, Grosse J, Hellmig O, Müntinga H, Schkolnik V, Wendrich T et al (2018) Space-borne Bose-Einstein condensation for precision interferometry. Nature 562(7727):391-395. https://doi.org/10.1038/s41586018-0605-1

Berceau P, Taylor M, Kahn J, Hollberg L (2016) Space-time reference with an optical link. Class Quantum Gravity 33(13):135007. https://doi.org/10.1088/0264-9381/33/13/135007

Bergeron H, Sinclair LC, Swann WC, Khader I, Cossel KC, Cermak M, Deschênes JD, Newbury NR (2019) Femtosecond time synchronization of optical clocks off of a flying quadcopter. Nat Commun 10(1):1819. https://doi.org/10.1038/s41467-019-09768-9

Bingham RJ, Knudsen P, Andersen O, Pail R (2011) An initial estimate of the North Atlantic steady-state geostrophic circulation from GOCE. Geophys Res Lett. https://doi.org/10.1029/ 2010GL045633

Bjerhammar A (1986) Relativistic geodesy. Technical report. National Oceanic and Atmospheric Administration, National Ocean Service 118, National Geodetic Survery 36

Bjerhammar A (1985) On a relativistic geodesy. Bull Géod 59(3):207220. https://doi.org/10.1007/BF02520327

Bock H, Jäggi A, Beutler G, Meyer U (2014) GOCE: precise orbit determination for the entire mission. J Geod 88(11):1047-1060. https://doi.org/10.1007/s00190-014-0742-8

Bondarescu R, Schärer A, Lundgren A, Hetényi G, Houliè N, Jetzer P, Bondarescu M (2015) Ground-based optical atomic clocks as a tool to monitor vertical surface motion. Geophys J Int 202(3):17701774. https://doi.org/10.1093/gji/ggv246

Brewer SM, Chen JS, Hankin AM, Clements ER, Chou CW, Wineland DJ, Hume DB, Leibrandt DR (2019) ${ }^{27} \mathrm{Al}^{+}$quantum-logic clock with a systematic uncertainty below $10^{-18}$. Phys Rev Lett 123:033201. https://doi.org/10.1103/PhysRevLett.123.033201

Brockmann JM, Zehentner N, Höck E, Pail R, Loth I, Mayer-Gürr T, Schuh WD (2014) EGM_TIM_RL05: an independent geoid with centimeter accuracy purely based on the GOCE mission. Geophys Res Lett 41(22):8089-8099. https://doi.org/10.1002/ 2014GL061904

Browne D, Bose S, Mintert F, Kim M (2017) From quantum optics to quantum technologies. Prog Quantum Electron 54:2-18. https:// doi.org/10.1016/j.pquantelec.2017.06.002

Bruinsma SL, Förste C, Abrikosov O, Lemoine JM, Marty JC, Mulet S, Rio MH, Bonvalot S (2014) ESA's satellite-only gravity field model via the direct approach based on all GOCE data. Geophys Res Lett 41(21):7508-7514. https://doi.org/10.1002/ 2014GL062045 
Carraz O, Siemes C, Massotti L, Haagmans R, Silvestrin P (2014) A spaceborne gravity gradiometer concept based on cold atom interferometers for measuring Earth's gravity field. Microgravity Sci Technol 26(3):139-145. https://doi.org/10.1007/s12217-0149385- $\mathrm{x}$

Delva P, Hees A, Wolf P (2017) Clocks in space for tests of fundamental physics. Space Sci Rev 212(3):1385-1421. https://doi.org/10. 1007/s11214-017-0361-9

Delva P, Denker H, Lion G (2019) Chronometric geodesy: methods and applications. Springer, Berlin, pp 25-85. https://doi.org/10.1007/ 978-3-030-11500-5_2

Delva P, Meynadier F, Le Poncin-Lafitte C, Laurent P, Wolf P (2012) Time and frequency transfer with a microwave link in the ACES/PHARAO mission. In: European frequency and time forum, pp 28-35. https://doi.org/10.1109/EFTF.2012.6502327

Denker H, Timmen L, Voigt C, Weyers S, Peik E, Margolis HS, Delva P, Wolf P, Petit G (2018) Geodetic methods to determine the relativistic redshift at the level of $10^{-18}$ in the context of international timescales: a review and practical results. J Geod 92(5):487-516. https://doi.org/10.1007/s00190-017-1075-1

Dobslaw H, Bergmann-Wolf I, Forootan E, Dahle C, Mayer-Gürr T, Kusche J, Flechtner F (2016) Modeling of present-day atmosphere and ocean non-tidal de-aliasing errors for future gravity mission simulations. J Geod 90(5):423-436. https://doi.org/10. 1007/s00190-015-0884-3

Douch K, Wu H, Schubert C, Müller J, Pereira dos Santos F (2018) Simulation-based evaluation of a cold atom interferometry gradiometer concept for gravity field recovery. Adv Space Res 61(5):1307-1323. https://doi.org/10.1016/j.asr.2017.12.005

Elsaka B, Raimondo JC, Brieden P, Reubelt T, Kusche J, Flechtner F, Iran Pour S, Sneeuw N, Müller J (2014) Comparing seven candidate mission configurations for temporal gravity field retrieval through full-scale numerical simulation. J Geod 88(1):31-43. https://doi.org/10.1007/s00190-013-0665-9

ESA (2014) GOCE standards. Technical report GO-TN-HPF-GS-0111, Issue 4.0

Förste C, Bruinsma S, Abrikosov O, Flechtner F, Marty JC, Lemoine JM, Dahle C, Neumayer H, Barthelmes F, König R, Biancale R (2014) EIGEN-6C4 - the latest combined global gravity field model including GOCE data up to degree and order 1949 of GFZ Potsdam and GRGS Toulouse. In: EGU general assembly conference abstracts, vol 16, p 3707

Gill P, Margolis H, Curtis A, Klein H, Lea S, Webster S, Whibberley P (2008) Optical atomic clocks for space. Technical report, National Physical Laboratory

Grotti J, Koller S, Vogt S, Häfner S, Sterr U, Lisdat C, Denker H, Voigt C, Timmen L, Rolland A, Baynes FN, Margolis HS, Zampaolo M, Thoumany P, Pizzocaro M, Rauf B, Bregolin F, Tampellini A, Barbieri P, Zucco M, Costanzo GA, Clivati C, Levi F, Calonico D (2018) Geodesy and metrology with a transportable optical clock. Nat Phys 14(5):437-441. https://doi.org/10.1038/s41567017-0042-3

Hachisu H, Fujieda M, Nagano S, Gotoh T, Nogami A, Ido T, Falke S, Huntemann N, Grebing C, Lipphardt B, Lisdat C, Piester D (2014) Direct comparison of optical lattice clocks with an intercontinental baseline of $9000 \mathrm{~km}$. Opt Lett 39(14):4072-4075. https://doi.org/ 10.1364/OL.39.004072

Hofmann-Wellenhof B, Moritz H (2006) Physical geodesy. Springer, Berlin

Ince ES, Pagiatakis SD (2016) Effects of space weather on GOCE electrostatic gravity gradiometer measurements. J Geod 90(12):13891403. https://doi.org/10.1007/s00190-016-0931-8

Kitching J, Knappe S, Donley EA (2011) Atomic sensors: a review. IEEE Sens J 11(9):1749-1758. https://doi.org/10.1109/JSEN. 2011.2157679
Koch KR (1999) Parameter estimation and hypothesis testing in linear models. Springer, Berlin. https://doi.org/10.1007/978-3-66203976-2

Koller SB, Grotti J, Vogt S, Al-Masoudi A, Dörscher S, Häfner S, Sterr U, Lisdat C (2017) Transportable optical lattice clock with $7 \times$ $10^{-17}$ uncertainty. Phys Rev Lett 118:073601. https://doi.org/10. 1103/PhysRevLett.118.073601

Kornfeld RP, Arnold BW, Gross MA, Dahya NT, Klipstein WM, Gath PF, Bettadpur S (2019) GRACE-FO: the gravity recovery and climate experiment follow-on mission. J Spacecr Rockets 56(3):931-951. https://doi.org/10.2514/1.A34326

Laurent P, Massonnet D, Cacciapuoti L, Salomon C (2015) The ACES/PHARAO space mission. CR Phys 16(5):540-552. https:// doi.org/10.1016/j.crhy.2015.05.002

Leute J, Huntemann N, Lipphardt B, Tamm C, Nisbet-Jones PBR, King SA, Godun RM, Jones JM, Margolis HS, Whibberley PB, Wallin A, Merimaa M, Gill P, Peik E (2016) Frequency comparison of ${ }^{171} \mathrm{Yb}^{+}$ion optical clocks at PTB and NPL via GPS PPP. IEEE Trans Ultrason Ferroelectr Freq Control 63(7):981-985. https:// doi.org/10.1109/TUFFC.2016.2524988

Lion G, Panet I, Wolf P, Guerlin C, Bize S, Delva P (2017) Determination of a high spatial resolution geopotential model using atomic clock comparisons. J Geod 91(6):597-611. https://doi.org/ 10.1007/s00190-016-0986-6

Lisdat C, Grosche G, Quintin N, Shi C, Raupach S, Grebing C, Nicolodi D, Stefani F, Al-Masoudi A, Dörscher S et al (2016) A clock network for geodesy and fundamental science. Nat Commun. https:// doi.org/10.1038/ncomms 12443

Lodewyck J (2019) On a definition of the SI second with a set of optical clock transitions. Metrologia 56(5):055009. https://doi.org/10. 1088/1681-7575/ab3a82

Ludlow AD, Boyd MM, Ye J, Peik E, Schmidt PO (2015) Optical atomic clocks. Rev Mod Phys 87:637-701. https://doi.org/10. 1103/RevModPhys.87.637

McGrew WF, Zhang X, Fasano RJ, Schäffer SA, Beloy K, Nicolodi D, Brown RC, Hinkley N, Milani G, Schioppo M, Yoon TH, Ludlow AD (2018) Atomic clock performance enabling geodesy below the centimetre level. Nature 564(7734):87-90. https://doi.org/10. 1038/s41586-018-0738-2

Mehlstäubler TE, Grosche G, Lisdat C, Schmidt PO, Denker H (2018) Atomic clocks for geodesy. Rep Prog Phys 81(6):064401. https:// doi.org/10.1088/1361-6633/aab409

Migliaccio F, Reguzzoni M, Batsukh K, Tino GM, Rosi G, Sorrentino F, Braitenberg C, Pivetta T, Barbolla DF, Zoffoli S (2019) MOCASS: A satellite mission concept using cold atom interferometry for measuring the Earth gravity field. Surv Geophys 40(5):1029-1053. https://doi.org/10.1007/s10712-019-09566-4

Müller J, Dirkx D, Kopeikin SM, Lion G, Panet I, Petit G, Visser P (2018) High performance clocks and gravity field determination. Space Sci Rev 214(1):5. https://doi.org/10.1007/s11214017-0431-z

Oelker E, Hutson RB, Kennedy CJ, Sonderhouse L, Bothwell T, Goban A, Kedar D, Sanner C, Robinson JM, Marti GE, Matei DG, Legero T, Giunta M, Holzwarth R, Riehle F, Sterr U, Ye J (2019) Demonstration of $4.8 \times 10^{-17}$ stability at $1 \mathrm{~s}$ for two independent optical clocks. Nat Photonics 13(10):714-719. https://doi.org/10. 1038/s41566-019-0493-4

Origlia S, Pramod MS, Schiller S, Singh Y, Bongs K, Schwarz R, AlMasoudi A, Dörscher S, Herbers S, Häfner S, Sterr U, Lisdat C (2018) Towards an optical clock for space: compact, highperformance optical lattice clock based on bosonic atoms. Phys Rev A 98:053443. https://doi.org/10.1103/PhysRevA.98.053443

Pail R, Bruinsma S, Migliaccio F, Förste C, Goiginger H, Schuh WD, Höck E, Reguzzoni M, Brockmann JM, Abrikosov O, Veicherts M, Fecher T, Mayrhofer R, Krasbutter I, Sansò F, Tscherning CC (2011) First GOCE gravity field models derived by three differ- 
ent approaches. J Geod 85(11):819-843. https://doi.org/10.1007/ s00190-011-0467-x

Pail R, Bingham R, Braitenberg C, Dobslaw H, Eicker A, Güntner A, Horwath M, Ivins E, Longuevergne L, Panet I, Wouters B, IUGG Expert Panel (2015) Science and user needs for observing global mass transport to understand global change and to benefit society. Surv Geophys 36(6):743-772. https://doi.org/10.1007/ s10712-015-9348-9

Petit G, Leute J, Loyer S, Perosanz F (2017) Sub $10^{-16}$ frequency transfer with IPPP: recent results. In: Joint conference of the European frequency and time forum and IEEE international frequency control symposium (EFTF/IFCS), pp 784-787. https://doi.org/10. 1109/FCS.2017.8089035

Petit G, Wolf P, Delva P (2014) Atomic time, clocks, and clock comparisons in relativistic spacetime: a review, vol 2, chap 7, pp 249-279

Plag HP, Altamimi Z, Bettadpur S, Beutler G, Beyerle G, Cazenave A, Crossley D, Donnellan A, Forsberg R, Gross R, Hinderer J, Komjathy A, Ma C, Mannucci A, Noll C, Nothnagel A, Pavlis E, Pearlman M, Poli P, Schreiber U, Senior K, Woodworth P, Zerbini S, Zuffada C (2009) The goals, achievements, and tools of modern geodesy. Springer, Berlin, pp 15-88. https://doi.org/10.1007/9783-642-02687-4_2

Rakholia A, Sugarbaker A, Black A, Kasevich M, Saif B, Luthcke S, Callahan L, Seery BD, Feinberg L, Mather JC, Keski-Kuha R (2017) Development of an atom interferometer gravity gradiometer for Earth sciences. In: APS division of atomic, molecular and optical physics meeting abstracts, APS meeting abstracts

Reguzzoni M, Sampietro D, Sansò F (2013) Global Moho from the combination of the CRUST2.0 model and GOCE data. Geophys J Int 195(1):222-237. https://doi.org/10.1093/gji/ggt247

Riehle F (2017) Optical clock networks. Nat Photonics 11(1):25-31. https://doi.org/10.1038/nphoton.2016.235

Rummel R, Yi W, Stummer C (2011) GOCE gravitational gradiometry. J Geod 85(11):777-790. https://doi.org/10.1007/s00190-0110500-0

Schuh WD (2003) The processing of band-limited measurements; filtering techniques in the least squares context and in the presence of data gaps. Space Sci Rev 108(1):67-78. https://doi.org/10.1023/ A:1026121814042

Schuldt T, Gohlke M, Sanjuan Munoz J, Abich K, Oswald M, Döringshoff K, Kovalchuk E, Peters A, Braxmaier C (2018) Optical clock technologies for future GNSS. In: Proceedings of the 31st International technical meeting of the Satellite Division of The Institute of Navigation (ION GNSS+ 2018), Miami, Florida, pp 857-860

Seiferle B, von der Wense L, Bilous PV, Amersdorffer I, Lemell C, Libisch F, Stellmer S, Schumm T, Düllmann CE, Pálffy A, Thirolf PG (2019) Energy of the 229th nuclear clock transition. Nature 573(7773):243-246. https://doi.org/10.1038/s41586-019-1533-4

Sheard BS, Heinzel G, Danzmann K, Shaddock DA, Klipstein WM, Folkner WM (2012) Intersatellite laser ranging instrument for the GRACE follow-on mission. J Geod 86(12):1083-1095. https://doi. org/10.1007/s00190-012-0566-3

Sinclair LC, Bergeron H, Swann WC, Khader I, Cossel KC, Cermak M, Newbury NR, Deschênes JD (2019) Femtosecond optical twoway time-frequency transfer in the presence of motion. Phys Rev A 99:023844. https://doi.org/10.1103/PhysRevA.99.023844

Snadden MJ, McGuirk JM, Bouyer P, Haritos KG, Kasevich MA (1998) Measurement of the Earth's gravity gradient with an atom interferometer-based gravity gradiometer. Phys Rev Lett 81:971974. https://doi.org/10.1103/PhysRevLett.81.971
Sorrentino F, Bodart Q, Cacciapuoti L, Lien YH, Prevedelli M, Rosi G, Salvi L, Tino GM (2014) Sensitivity limits of a Raman atom interferometer as a gravity gradiometer. Phys Rev A 89:023607. https://doi.org/10.1103/PhysRevA.89.023607

Takano T, Takamoto M, Ushijima I, Ohmae N, Akatsuka T, Yamaguchi A, Kuroishi Y, Munekane H, Miyahara B, Katori H (2016) Geopotential measurements with synchronously linked optical lattice clocks. Nat Photonics 10:662-666. https://doi.org/10.1038/ nphoton.2016.159

Tapley BD, Bettadpur S, Ries JC, Thompson PF, Watkins MM (2004) GRACE measurements of mass variability in the Earth system. Science 305(5683):503-505. https://doi.org/10.1126/ science. 1099192

Tino G, Cacciapuoti L, Bongs K, Bordé C, Bouyer P, Dittus H, Ertmer W, Görlitz A, Inguscio M, Landragin A, Lemonde P, Lammerzahl C, Peters A, Rasel E, Reichel J, Salomon C, Schiller S, Schleich W, Sengstock K, Sterr U, Wilkens M (2007) Atom interferometers and optical atomic clocks: new quantum sensors for fundamental physics experiments in space. Nucl Phys B Proc Suppl 166:159_ 165. https://doi.org/10.1016/j.nuclphysbps.2006.12.061

Torge W, Müller J (2012) Geodesy. De Gruyter, Berlin

Touboul P, Métris G, Sélig H, Traon OL, Bresson A, Zahzam N, Christophe B, Rodrigues M (2016) Gravitation and geodesy with inertial sensors, from ground to space. Aerospace Lab. https://doi. org/10.12762/2016.AL12-11

Trimeche A, Battelier B, Becker D, Bertoldi A, Bouyer P, Braxmaier C, Charron E, Corgier R, Cornelius M, Douch K, Gaaloul N, Herrmann S, Müller J, Rasel E, Schubert C, Wu H, Pereira dos Santos F (2019) Concept study and preliminary design of a cold atom interferometer for space gravity gradiometry. Class Quantum Gravity 36(21):215004. https://doi.org/10.1088/1361-6382/ab4548

Vermeer M (1983) Chronometric levelling. Technical report, Finnish Geodetic Institute

Wu H (2016) Gravity field recovery from GOCE observations. PhD thesis, Nr. 324, Institute of Geodesy, Leibniz University of Hannover, Germany

Wu H, Müller J, Lämmerzahl C (2018) Clock networks for height system unification: a simulation study. Geophys J Int 216(3):1594 1607. https://doi.org/10.1093/gji/ggy508

Yu N, Kohel J, Kellogg J, Maleki L (2006) Development of an atom-interferometer gravity gradiometer for gravity measurement from space. Appl Phys B 84(4):647-652. https://doi.org/10.1007/ s00340-006-2376-x 